\title{
F-18 FDG PET/CT in pulmonary artery sarcoma: clinical vignette
}

Stjepan Hmelik ${ }^{1,2}$, Margareta Dobrenić ${ }^{3,4}$, Dražen Huić3, 4

${ }^{1}$ General Hospital Varazdin, Varazdin, Croatia

2University Hospital Centre Zagreb, Zagreb, Croatia

${ }^{3}$ University Hospital Centre Zagreb, Zagreb, Croatia

${ }^{4}$ School of Medicine Zagreb, Croatia, Zagreb, Croatia

[Received 19 IX 2017; Accepted 28 XI 2017]

\section{Introduction}

Pulmonary artery sarcomas (PASs) are extremely rare malignant tumors that are usually misdiagnosed as pulmonary embolisms because of their similar symptoms and similar appearance on CT (computed tomography) scans as intraluminal filling defects in the pulmonary arteries. Due to the fact that PASs are fluorodeoxyglucose F-18 (F-18 FDG) avid, F-18 FDG PET/CT (positron emission tomography/CT) scan is a useful imaging tool for differentiating malignant intraluminal mass of PAS from the intraluminal mass in pulmonary artery embolism, as this case shows.

\section{Case report}

We report a case of a 60-year-old woman presented with a 6-month history of chest pain, dyspnea on exertion, non-productive cough and weight loss. The initial CT pulmonary artery angiography showed extensive intraluminal mass in the pulmonary trunk and left pulmonary artery, diagnosed as massive pulmonary embolism. Since there was no clinical improvement after anticoagulant therapy, CT pulmonary angiography was repeated (Figure 1), and with no change observed in the intraluminal filling defect in pulmonary trunk, the possibility of tumor was raised. For further evaluation of a possible malignancy, F-18 FDG PET/CT was performed. It showed increased FDG uptake, suspicious for an aggressive tumor, in the intraluminal lesion of the pulmonary trunk and along the wall of the left pulmonary artery (Figure 2). There was no extrathoracic abnormality seen on PET/CT scan. Histopathological finding after complete pulmonary artery resection and left pneumonectomy showed a $9 \mathrm{~cm}$ long high grade undifferentiated pleomorphic sarcoma. No metastases were found in the parenchyma of the left lung. Three months after diagnosis, the patient was doing well and was receiving adjuvant chemotherapy treatment consisting of gemcitabine/docetaxel combination.

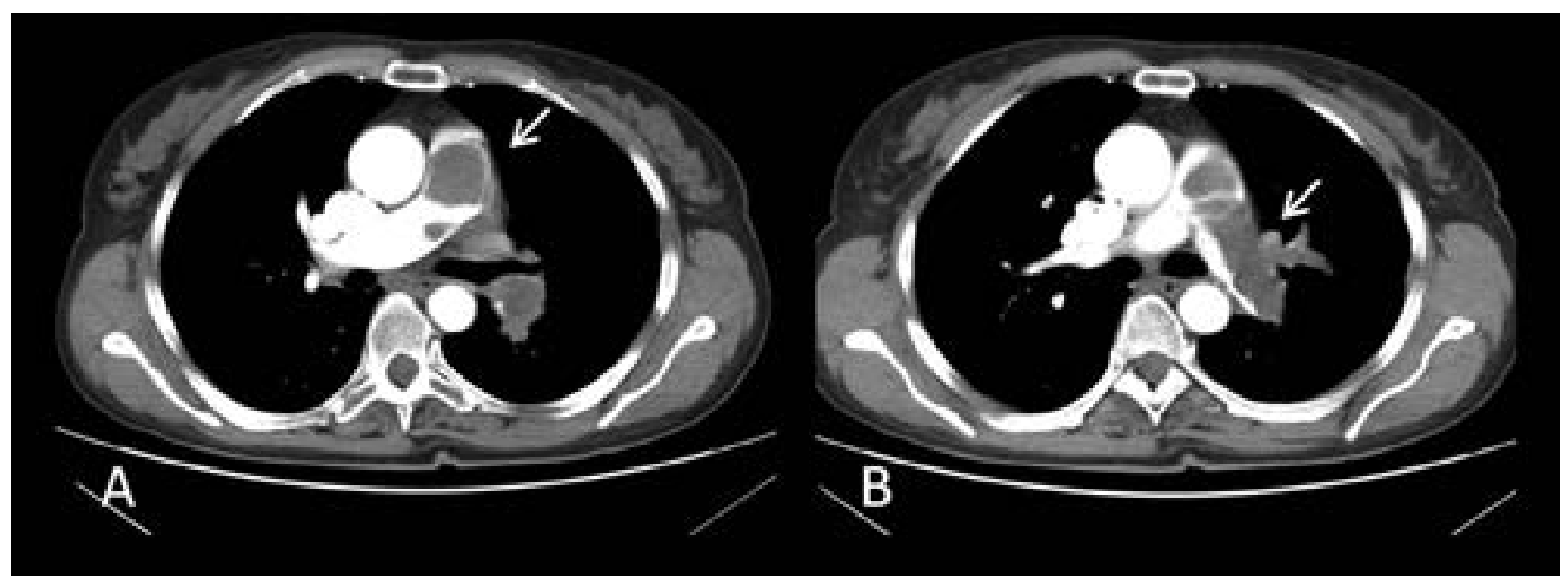

Figure 1. Repeated CT pulmonary angiography showed extensive intraluminal filling defect in the pulmonary trunk stretching from pulmonary valve to the bifurcation (A), with extension into the left pulmonary artery and its lobar and segmental branches (B), possible intraluminal tumor

Correspondence to: Stjepan Hmelik, General Hospital Varazdin, I.

Mestrovica b.b., 42000, Varazdin, Croatia; University Hospital Centre Zagreb,

Kispaticeva 12, 10000, Zagreb, Croatia, e-mail: hmelik88@gmail.com 


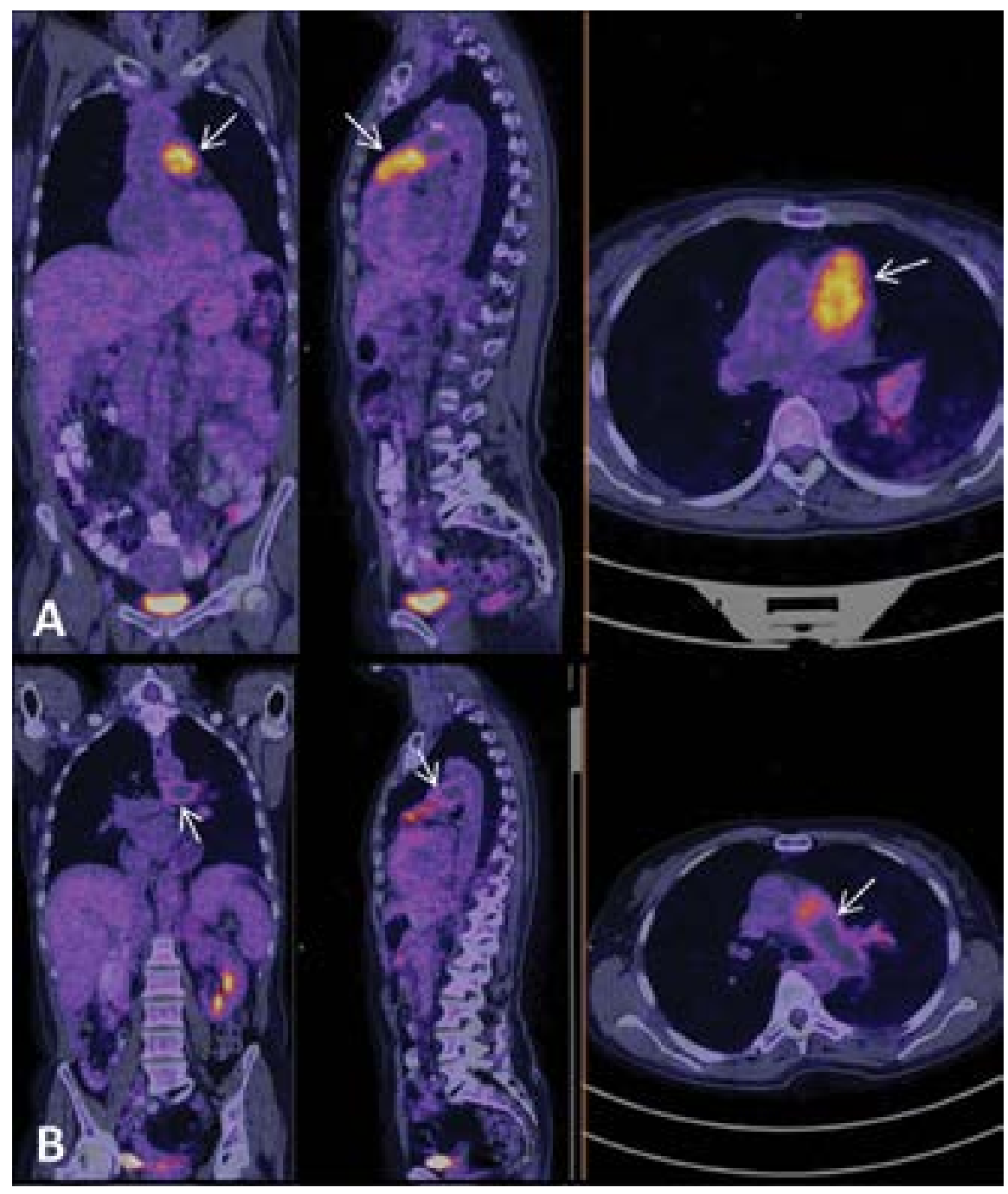

Figure 2. F-18 FDG PET/CT. Increased FDG uptake seen in an intraluminal lesion of the pulmonary trunk from pulmonary valve to bifurcation (A, SUVmax 10). Increased FDG uptake seen along the wall of the left pulmonary artery and its lobar branches (B, SUVmax 5), without intraluminal accumulation in that area

\section{Conclusion}

Pulmonary artery sarcomas are rare and aggresive malignant tumors with less then 400 individual cases reported in the litererature. Due to similar clinical and radiological presentation they are usually misdiagnosed as pulmonary embolisms. We report a case of pulmonary artery sarcoma that was appropriately diagnosed with the aid of
F-18 FDG PET/CT imaging. PET/CT has shown the ability to differentiate pulmonary embolism and PAS before invasive procedures and at the same time enable the proper staging of the malignancy and help the surgeons to detect the extent of viable tumor and plan the appropriate therapy. Also, this case demonstrates the importance of maintaining a suspicion of PAS in those patients diagnosed as having PE whose symptoms are not improving with anticoagulation therapy. 